\title{
Rv0954 Is a Member of the Mycobacterial Cell Division Complex
}

\author{
Ruojun Wang ${ }^{\dagger}$ and Sabine Ehrt* \\ Department of Microbiology and Immunology, Weill Cornell Medical College, New York, NY, United States
}

OPEN ACCESS

Edited by:

Hari S. Misra,

Bhabha Atomic Research Centre

(BARC), India

Reviewed by:

Vinay Kumar Nandicoori,

National Institute of Immunology (NII),

India

Murty Madiraju,

The University of Texas Health

Science Center at Tyler, United States

${ }^{*}$ Correspondence:

Sabine Ehrt

sae2004@med.cornell.edu

${ }^{\dagger}$ Present address:

Ruojun Wang,

Department of Molecular Biology,

Princeton University, Princeton, NJ,

United States

Specialty section:

This article was submitted to

Microbial Physiology and Metabolism,

a section of the journal

Frontiers in Microbiology

Received: 05 November 2020

Accepted: 15 March 2021

Published: 20 April 2021

Citation:

Wang $R$ and Ehrt S (2021)

Rv0954 Is a Member of the

Mycobacterial Cell Division Complex.

Front. Microbiol. 12:626461.

doi: 10.3389/fmicb.2021.626461
Proper control of cell division in the intracellular pathogen Mycobacterium tuberculosis is central to its growth, survival, pathogenesis, and resistance to antibiotics. Nevertheless, the divisome components and mechanisms by which mycobacteria regulate their cell cycle are not entirely understood. Here we demonstrate that the previously uncharacterized Rv0954 protein localizes to the mid-cell during cell division and interacts with the division-related proteins LamA, PbpA, and PknH. Deletion of rv0954 did not result in alterations in cell morphology or sensitivity to cell wall-targeting antibiotics but transposon mutagenesis demonstrated genetic interactions with genes related to cell division. This work suggests that Rv0954 participates in cell division and reveals potential components of the mycobacterial divisome for future investigation.

Keywords: Mycobacterium tuberculosis, cell division, divisome, phosphorylation, Tnseq

\section{INTRODUCTION}

Our knowledge on how one bacterium becomes two has advanced significantly since Y. Hirota, A. Ryter, and F. Jacob first characterized a collection of thermosensitive Escherichia coli (E. coli) cell division mutants about half a century ago (Hirota et al., 1968). In the pathogenic mycobacterial species, Mycobacterium tuberculosis (Mtb) and Mycobacterium leprae, cell division control is an integral part of host-pathogen interactions and may contribute to disease outcomes (Kieser and Rubin, 2014). However, there are many remaining questions to answer and new components to discover in mycobacterial cell division.

Cell division in mycobacteria differs from model rod-shaped bacteria, E. coli and Bacillus subtilis, in several aspects (Kieser and Rubin, 2014; Baranowski et al., 2019). First, mycobacteria have a complex cell wall consisting of peptidoglycan, arabinogalactan, and mycolic acids (Baranowski et al., 2019). Splitting and synthesizing this highly sophisticated cell envelope at the division site poses a unique challenge for mycobacteria. Second, mycobacteria elongate at the poles in contrast to lateral elongation in the model organisms (Meniche et al., 2014; Cameron et al., 2015). As the division site matures into an elongation site, mycobacteria need to ensure both events are coordinated in time and space by tightly regulating the cell division complex (divisome) and the elongation machinery (Baranowski et al., 2019). Third, model bacteria divide precisely at mid-cell, creating two daughter cells of near-identical length; mycobacteria, on the other hand, grow and divide asymmetrically, leading to daughter cells of varying lengths (Aldridge et al., 2012; Santi et al., 2013). This heterogeneity is promoted by LamA and may be advantageous for the mycobacterial 
population to survive host stresses and antibiotics treatment (Rego et al., 2017). Mycobacteria's unusual cell division features likely explain why they lack homologs of many divisome components found in model bacteria and instead encode speciesspecific factors (Kieser and Rubin, 2014; Baranowski et al., 2019).

Genes with related functions are often located in the same operon allowing co-regulation. The Mtb genome contains many such loci; for example, the $r v 0014 c$ - rv0018c region encodes PknA (Rv0015c) and PknB (Rv0014c), two serine/threonine protein kinases that regulate cell division, and their cognate phosphatase, PstP (Rv0018c). The same region also contains $p b p A(r v 0016 c)$ and $\operatorname{rod} A(r v 0017 c)$, which encode peptidoglycan synthases involved in septum formation during cell division (Sureka et al., 2010). Since $r v 0954$ is in the same operon with perM, which encodes a division protein (Goodsmith et al., 2015; Wang et al., 2019), we hypothesized that Rv0954, a previously uncharacterized protein, may function in cell division or cell wall biosynthesis.

Like perM, rv0954 is one of 219 mycobacterial "core" genes conserved among mycobacterial species and without homologs in other bacteria (Marmiesse et al., 2004). Saturating transposon mutagenesis predicted rv0954 to be non-essential for growth of Mtb in rich medium (DeJesus et al., 2017), and topology prediction indicated that Rv0954 contains four transmembrane helixes (TMHs) flanked by cytoplasmic N- and C- termini (Supplementary Figure 1). The C- terminal half of Rv0954 (aa $155-303)$ is rich in proline (18\%) and glutamine residues (15\%), which often form extended and rigid structures (Williamson, 1994) and may allow Rv0954 to protrude into the cytoplasm for some distance. Here, we examined Rv0954's localization during the cell cycle, identified its physical interaction partners, and discovered candidate genes that may encode proteins with overlapping functions with Rv0954. Our results support a role for Rv0954 in cell division.

\section{MATERIALS AND METHODS}

\section{Bacterial Culture Conditions}

Mycobacterium smegmatis (Msm) $\mathrm{mc}^{2} 155$ and derivative strains were cultured in Middlebrook 7H9 medium (BD Difco) containing $0.2 \%$ glycerol and $0.05 \%$ Tween 80 or Middlebrook 7H10 agar (BD Difco) containing 0.5\% glycerol. Mtb H37Rv and derivative strains were cultured in Middlebrook 7H9 medium containing $0.2 \%$ glycerol, $0.2 \%$ dextrose, $0.5 \%$ BSA (Roche), $0.05 \%$ Tween 80 or tyloxapol, and $0.085 \% \mathrm{NaCl}$ or Middlebrook $7 \mathrm{H} 10$ agar containing 10\% OADC supplement (BD) and 0.5\% glycerol. Strains bearing antibiotic resistance cassettes were cultured with hygromycin $(50 \mu \mathrm{g} / \mathrm{ml})$, zeocin $(25 \mu \mathrm{g} / \mathrm{ml}$ ), kanamycin $(25 \mu \mathrm{g} / \mathrm{ml})$ or streptomycin $(20 \mu \mathrm{g} / \mathrm{ml})$. Growth curves in acidic and low- $\mathrm{Mg}^{2+}$ conditions were performed as previously described (Goodsmith et al., 2015; Wang et al., 2019).

\section{Mutant Construction}

The Mtb $\Delta r v 0954$ mutant was constructed by allelic exchange using a recombineering approach as previously described (Gee et al., 2012). The $\Delta r v 0954$ strain was complemented by introducing a copy of $r v 0954$ expressed from the $h s p 60$ promoter into the Mtb genome. We generated the msmeg_5518 single deletion mutant $\left(\Delta m s m e g \_5518\right.$-per $M_{\mathrm{msm}}:$ per $\left.M_{\mathrm{mtb}}\right)$ by first integrating per $M_{\mathrm{mtb}}$ to the attL5 site, then deleting the operon containing msmeg_5518 and per $M_{\mathrm{msm}}$. The rv0954 phospho mutants were constructed by integrating a copy of phosphoablative or phospho-mimetic Rv0954 fused with C-terminal GFP tags expressed from $p 38$ promoters into the genome.

\section{High-Resolution Microscopy}

Microscopy imaging was performed using the same methods and equipment as described (Botella et al., 2017). The Mtb samples were fixed with $4 \%$ paraformaldehyde for $4 \mathrm{~h}$ before removal from BSL-3 containment. Single-cell suspensions were prepared by centrifugation at $800 \mathrm{rpm}$ for $10 \mathrm{~min}$. After spreading on a soft agar pad, the bacteria were visualized using a microscope with appropriate filter sets. For the time-lapse microscopy experiment, we place a drop of Msm single-cell culture $(5 \sim 10 \mu \mathrm{l})$ to a glassbottom microwell dish (MatTek, $35 \mathrm{~mm}, 14 \mathrm{~mm}$ microwell). $1 \%$ low melting point agarose (UltraPure, Invitrogen) prepared in 7H9 broth was carefully added on top of the bacterial drop. Agarose was left at room temperature to allow solidification before microscopy. We captured snapshots every $15 \mathrm{~min}$ and analyzed images with ImageJ (Abramoff et al., 2003).

\section{Co-immunoprecipitation}

We harvested $150 \mathrm{ml}$ of log-phase Mtb cultures and incubated whole-cell lysates with $1 \%$ DDM for $2 \mathrm{~h}$ before overnight incubation with anti-Flag affinity gel (Sigma) at $4^{\circ} \mathrm{C}$. Beads were then washed with lysis buffer $(50 \mathrm{mM}$ Tris- $\mathrm{HCl}, 50 \mathrm{mM} \mathrm{NaCl}, \mathrm{pH}$ 7.4) and eluted by incubation with $100 \mathrm{ng} / \mu \mathrm{l}$ Flag peptide with gentle rotation for $1 \mathrm{~h}$ at $4^{\circ} \mathrm{C}$.

To confirm physical interactions, we harvested $30 \mathrm{ml} \mathrm{Msm}$ cultures for whole-cell protein lysates, which we incubated with $50 \mu \mathrm{l}$ anti-GFP mAb-magnetic beads (MBL) overnight shaking at $4^{\circ} \mathrm{C}$. After incubation, beads were collected using a DynaMagMagnet 2 rack (Thermo Fisher Scientific) and washed three times with lysis buffer. Proteins bound to beads were eluted by boiling in Laemmli sample buffer for $10 \mathrm{~min}$ and analyzed by immunoblotting.

\section{Antibiotic Susceptibility Assays}

Mtb was grown to early log phase and diluted to an OD of 0.005 in regular $7 \mathrm{H} 9$ medium containing $0.2 \%$ glycerol, $0.2 \%$ dextrose, $0.5 \%$ BSA, $0.05 \%$ Tween 80 , and $0.085 \% \mathrm{NaCl}$. Bacteria were then exposed to two-fold dilutions of compounds, and all wells contained 1\% DMSO. We recorded MIC as the minimum concentration at which growth was inhibited by at least $90 \%$, compared to controls after approximately 14 days.

\section{Phosphorylation Assay and Site Identification}

We incubated $20 \sim 40 \mu \mathrm{g}$ whole-cell protein lysates with $10 \mathrm{U}$ of alkaline phosphatase (Fast AP, Thermo Scientific) for $1 \mathrm{~h}$ at $37^{\circ} \mathrm{C}$. The same reactions without adding phosphatase served as controls. The samples were then boiled in Laemmli sample buffer 
and resolved on SDS-PAGE for western blotting. To identify Rv0954 phosphorylation sites, we enriched Rv0954-Flag from Mtb whole-cell lysates by pulldown. Eluates were resolved on SDS-PAGE, and gel pieces at the correct molecular weight range were collected for mass spectrometry identification.

\section{Transposon Library Construction}

The Mtb transposon libraries were constructed by himar1 mutagenesis as previously described (Abramoff et al., 2003). Briefly, approximately $5 \times 10^{10} \mathrm{CFU}$ of Mtb were incubated with $10^{11} \mathrm{PFU}$ of MycoMarT7 phage for $4 \mathrm{~h}$ at $37^{\circ} \mathrm{C}$. The cultures were collected, washed with $7 \mathrm{H} 9$ medium containing $0.05 \%$ Tween 80 , and cultured on $7 \mathrm{H} 9$ agar (7H9 medium containing $1.5 \%$ bacto agar, $10 \%$ OADC, $0.5 \%$ glycerol, $0.05 \%$ Tween 80 , and 25 $\mu \mathrm{g} / \mathrm{ml}$ kanamycin) for about 20 days at $37^{\circ} \mathrm{C}$. Both WT and $\Delta r v 0954$ libraries have TA dinucleotide site coverages of around
$50 \%$ by Illumina sequencing. Sequencing and data analysis were performed as previously described (Xu et al., 2017).

\section{RESULTS}

\section{Rv0954 Accumulates at the Mid-Cell Before Septum Formation and Persists Until Cell Constriction}

We examined Rv0954's localization by live-cell imaging using a fluorescent reporter Msm strain. Rv0954 localizes to the membrane in the pre-divisional cells, followed by its accumulation at the mid-cell that lasts until cell constriction (Figure 1). This localization pattern within the cell cycle suggests a role in cell division.

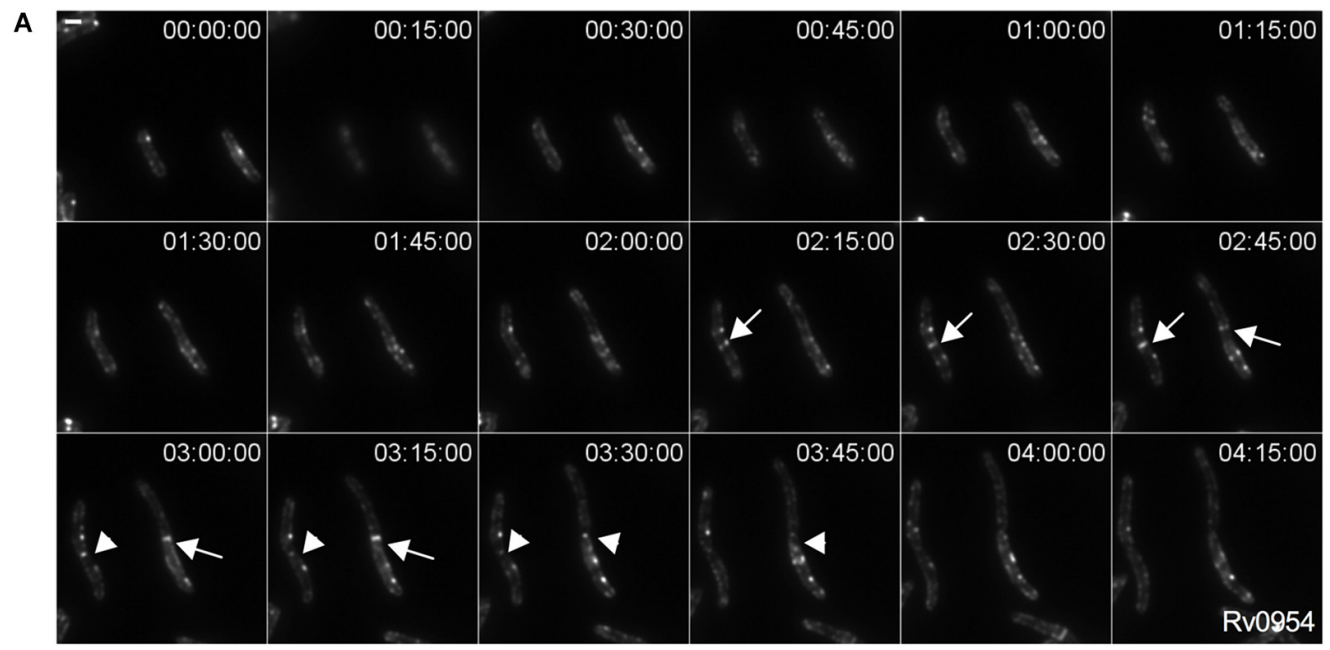

B

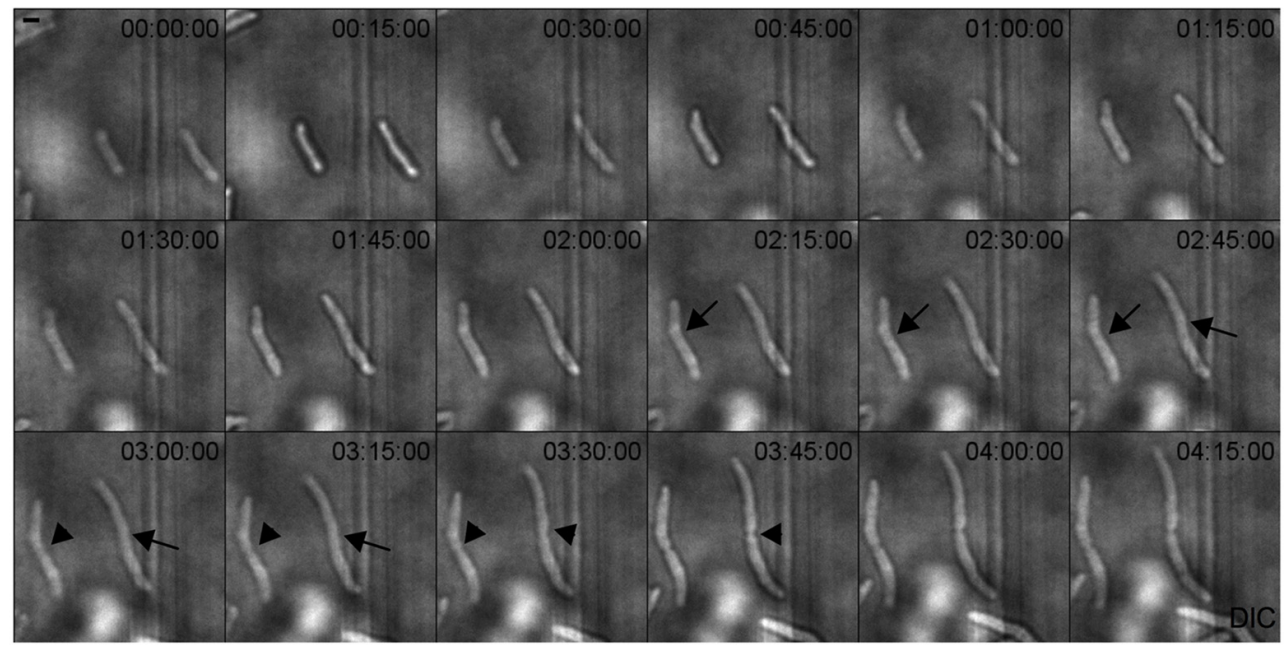

FIGURE 1 | Rv0954 localizes to the mid-cell during cell division. Representative image series from a time-lapse movie of replicating Msm that constitutively express Rv0954-GFP over $4 \mathrm{~h}$ and 15 min. Arrows point to mid-cell localization of Rv0954 before cell constriction, and arrowheads point to Rv0954 in cells undergoing constriction. Numbers in the upper right corner indicate time. Scale bar, $1 \mu \mathrm{m}$. (A) GFP channel. (B) Transmitted-light snapshots. 
We next compared the timing of Rv0954's mid-cell localization to known markers of the cell cycle (Figure 2A). FtsZ is one of the earliest divisome components to assemble at the midcell, marking divisome formation (Kieser and Rubin, 2014). Snapshots and quantification of Msm cells containing septal FtsZ bands while lacking mid-cell Rv0954 signals indicates that Rv0954 localizes to the mid-cell after the initiation of divisome assembly (Figures 2B,E). The fluorescent dye FM5-95 stains the cytoplasmic membrane, and its mid-cell signal is indicative of septum formation. In 7.5\% cells Rv0954 signals appeared at the mid-cell before septal membranes were visible (Figures 2C,E), indicating that Rv0954's mid-cell localization precedes septum formation. The mid-cell localization of Wag31 coincides with septum closure, which results in the cytoplasmic compartmentalization of future daughter cells (Santi et al., 2013). Co-localization of Rv0954 and Wag31 at the mid-cell suggested that Rv0954 remains at the septum when cytokinesis is completed (Figures 2D,E). Of particular interest are the cells exhibiting 'V-snapping' morphology (Figure 2D, top two panels). Consistent with its role in organizing polar elongation, Wag31 associated with the newly formed poles of future daughter cells (Santi et al., 2013; Kieser and Rubin, 2014). In contrast, Rv0954 localized to the $\mathrm{V}$ shape's inner edge, where the septum is not entirely resolved, perhaps to participate in the final steps
A

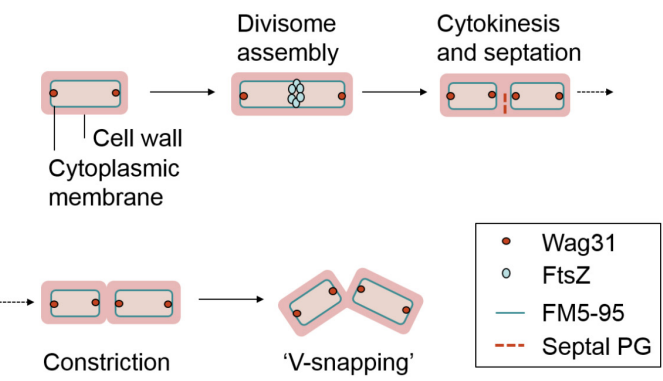

C

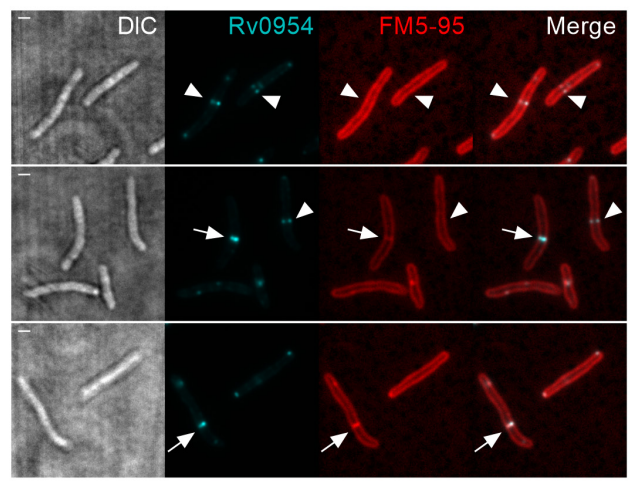

E

\begin{tabular}{|c|c|c|c|}
\hline Cell division markers & $\begin{array}{c}\text { \% bacteria with only } \\
\text { Rv0954 at mid-cell }\end{array}$ & $\begin{array}{c}\text { \% bacteria with only the } \\
\text { cell division marker at } \\
\text { mid-cell }\end{array}$ & $\begin{array}{c}\text { \% bacteria with both } \\
\text { Rv0954 and a cell division } \\
\text { marker at mid-cell }\end{array}$ \\
\hline FtsZ $(n=126)$ & 0.0 & 31.0 & 54.0 \\
\hline FM5-95 $(n=295)$ & 7.5 & 0.0 & 13.9 \\
\hline Wag31 $(n=71)$ & 29.6 & 0.0 & 46.5 \\
\hline
\end{tabular}

FIGURE 2 | Mid-cell localization of Rv0954 relative to markers of cell division events. (A) Schematic of division markers in different stages of cell division. (B-D) Representative microscopy images of replicating Msm expressing (B) FtsZmsm-GFP and Rv0954-mCherry (C) Rv0954-GFP (D) Rv0954-GFP and Wag31 $\mathrm{mtb}$-mCherry constitutively. (B) Arrowheads point to the presence of FtsZ signals and the absence of Rv0954 signals at the mid-cell. Arrows point to the colocalization of FtsZ and Rv0954. (C) Replicating Msm stained with FM5-95 to label the cytoplasmic membrane. Arrowheads point to the presence of Rv0954 signals and the absence of septal FM5-95 staining. Arrows point to the overlapping of Rv0954 and FM5-95 signals at the mid-cell. (D) White arrowheads point to the presence of Rv0954 and the absence of Wag31 mtb at the mid-cell. White arrows point to the mid-cell localization of both Rv0954 and Wag31 mtb. Yellow arrows point to Msm undergoing constriction. Scale bar, $1 \mu \mathrm{m}$. (E) Quantification of bacteria with Rv0954 and respective cell division markers at mid-cell. $N$ indicates the number of bacteria quantified. 
of cell separation. Overall, these co-localization experiments demonstrate that the mid-cell localization of Rv0954 precedes septum formation and lasts until cell constriction.

\section{Rv0954 Interacts With Several Proteins Involved in Cell Division and Cell Wall Biosynthesis}

Mycobacterial cell division is mediated by a large protein complex termed 'the divisome' (Kieser and Rubin, 2014). To identify protein interactors, we immunoprecipitated Rv0954 from Mtb lysates, followed by mass spectrometry (Figure 3 and Table 1). The 19 identified interactors included a serine/threonine protein kinase involved in cell division regulation: $\mathrm{PknH}$ (Sharma et al., 2006; Zheng et al., 2007) and two other known divisome proteins: LamA (also known as MmpS3) and PbpA. LamA is a septallocalizing protein that inhibits cell elongation from the new pole, and $\mathrm{PbpA}$ is a transpeptidase that localizes to both the septum and poles (Rego et al., 2017; Viswanathan et al., 2017; Arora et al., 2018). We confirmed the interaction between Rv0954 and $\mathrm{PknH}$ in Msm by co-immunoprecipitation (Supplementary Figure 2). The interaction of Rv0954 with these three proteins further supports its involvement in cell division.

\section{Deletion of rv0954 in Mtb Did Not Result in Apparent Alterations in Cell Morphology, Growth, or Susceptibility to Cell Wall-Targeting Compounds}

Rv0954 is predicted to be non-essential in Mtb (DeJesus et al., 2017); therefore, we generated a deletion mutant (Supplementary Figure 3). Cells lacking Rv0954 showed no apparent alterations in cell morphology or septum formation (Figure 4A). Deleting perM, which codes for a cell division component and is in an operon with rv0954, led to growth defects

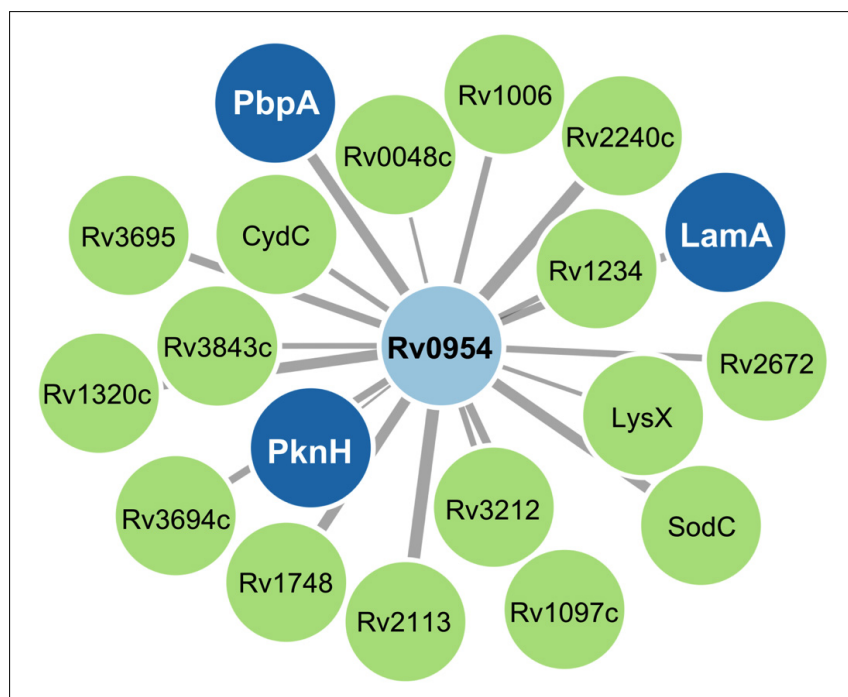

FIGURE 3 | Network map of Rv0954 interacting partners. Cell division-related proteins are marked in blue.
TABLE 1 | Identification of protein interaction partners of Rv0954 in Mtb by mass spectrometry.

\begin{tabular}{|c|c|c|c|}
\hline Rv\# & Gene & Annotation & $\begin{array}{l}\text { Sum total } \\
\text { spectrum count }\end{array}$ \\
\hline Rv1266c* & pknH & Serine/threonine-protein kinase $\mathrm{H}$ & 31 \\
\hline Rv0048c* & & Possible membrane protein & 20 \\
\hline Rv1640c* & lysX & Lysyl-tRNA synthetase & 17 \\
\hline $\mathrm{Rv} 3843 c^{*}$ & & $\begin{array}{l}\text { Probable conserved transmembrane } \\
\text { protein }\end{array}$ & 13 \\
\hline Rv1234* & & Transmembrane protein & 12 \\
\hline Rv3212* & & Conserved alanine valine rich protein & 12 \\
\hline Rv1620c* & cydC & $\begin{array}{l}\text { Thiol reductant } \mathrm{ABC} \text { exporter CydC } \\
\text { subunit }\end{array}$ & 12 \\
\hline Rv2672* & & Possible secreted protease & 11 \\
\hline Rv3694c* & & Transmembrane protein & 10 \\
\hline Rv1006 & & Hypothetical protein & 10 \\
\hline Rv1097c* & & $\begin{array}{l}\text { glycine and proline rich membrane } \\
\text { protein }\end{array}$ & 9 \\
\hline Rv3695* & & Hypothetical protein & 9 \\
\hline $\operatorname{Rv} 2198 c^{*}$ & $\begin{array}{l}\text { mmpS3/ } \\
\text { lamA }\end{array}$ & Divisome complex (Rego et al., 2017) & 9 \\
\hline Rv1748* & & Hypothetical protein & 7 \\
\hline Rv0016c* & $p b p A$ & Penicillin-binding protein A & 7 \\
\hline Rv0432 & sodC & Periplasmic superoxide dismutase & 7 \\
\hline Rv1320c* & & Adenylate cyclase & 7 \\
\hline Rv2240c* & & Hypothetical protein & 6 \\
\hline Rv2113* & & Integral membrane protein & 6 \\
\hline
\end{tabular}

Co-IP of Rv0954 from whole-cell lysates of Mtb WT:rv0954-flag. Whole-cell lysates of Mtb containing an empty vector or expressing Rv0060-Flag served as controls. We filtered out the non-specific binding peptides by setting the filter of "Total Spectrum Count" of each replicate to " $<4$ " in the Rv0060-Flag control samples, "<2" in the vector control samples, and ">2" in the Rv0954-Flag samples. The hits were then ranked based on the "Sum Total Spectrum Count" of the Rv0954Flag samples. The "Sum Total Spectrum Count" is the sum of two independent biological replicates. Annotations of each hit are adapted from PATRIC (https:// www.patricbrc.org/) and FLUTE (http://orca2.tamu.edu/U19/). Hits with predicted transmembrane domains are marked with *.

in acidic $\mathrm{pH}$ and in reduced $\mathrm{Mg}^{2+}$; thus, we tested $\Delta r v 0954$ in the two conditions (Goodsmith et al., 2015; Wang et al., 2019). Acidification slowed Mtb growth, but the $\Delta r v 0954$ mutant showed no growth defect relative to WT Mtb (Figure 4B). The $\Delta r v 0954$ mutant also replicated comparably to WT in medium with reduced $\mathrm{Mg}^{2+}$ (Figure 4C). Moreover, $\Delta r v 0954$ tolerated cell envelope targeting compounds similarly to WT (Table 2). These results indicate that the deletion of $r v 0954$ does not result in significant defects in cell division or cell wall biosynthesis.

\section{Transposon Sequencing (Tnseq) Experiments Revealed rv0954's Genetic Interactions With Genes Encoding Integral Membrane Proteins}

The lack of phenotypes associated with the deletion of rv0954 in Mtb suggested redundancy in the genome, as is the case for many other cell division components in bacteria, including mycobacteria (Kieser and Rubin, 2014). We expect genes of overlapping functions with $r v 0954$ to be more critical for Mtb 


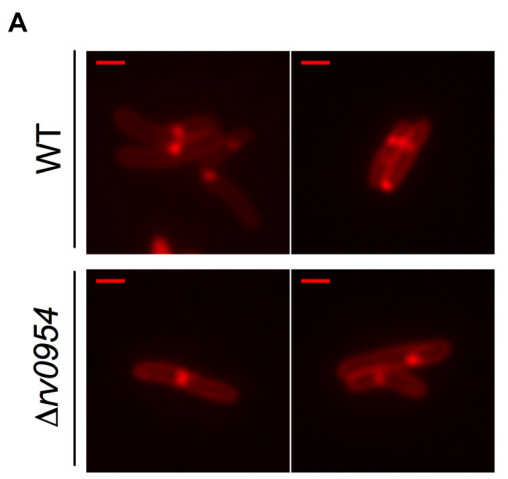

C WT

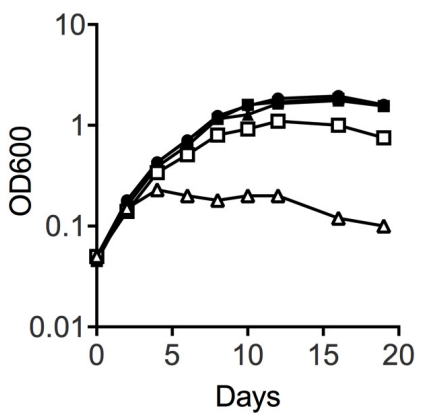

B

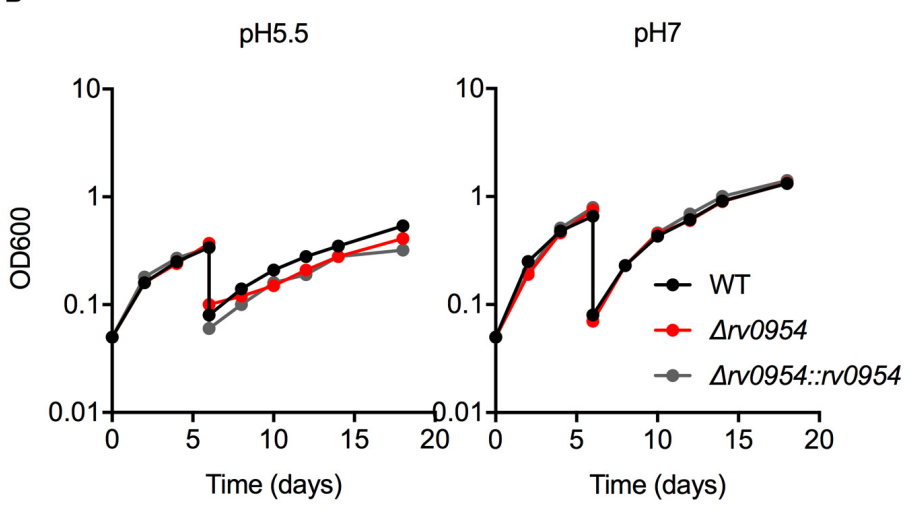

$\Delta r 0954$

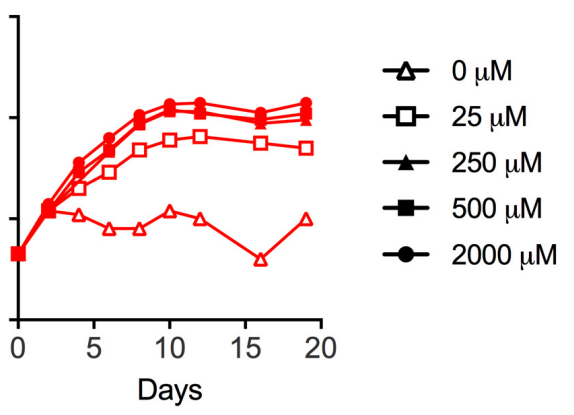

FIGURE 4 | Characterization of Mtb $\Delta r$ 0954. (A) Morphology of replicating WT and $\Delta r v 0954$ cultured in 7H9 broth. The bacterial cells were collected and stained with FM5-95 before imaging. Scale bar, $1 \mu \mathrm{m}$. (B) Growth curves of Mtb strains sequentially cultured in $7 \mathrm{H} 9$ medium adjusted to $\mathrm{pH} 5.5$ or $\mathrm{pH}$. The cultures were diluted in the same medium on day 6. (C) Growth curves of Mtb incubated in chelated Sauton's medium supplemented with Mg²+ at the indicated concentrations.

growth and survival when rv0954 is missing, and therefore transposon mutants of such genes should be underrepresented in the $\Delta r v 0954$ mutant relative to WT Mtb.

Four of the top 18 hits underrepresented in the $\Delta r v 0954$ mutant (Table 3) were involved in either cell division or cell wall biosynthesis. PimE (encoded by rv1159) is a mannosyltransferase involved in the biosynthesis of phosphatidylinositol mannosides (PIMs), glycolipids in the cell wall of mycobacteria (Morita et al., 2006; Crellin et al., 2008). The deletion of pimE in Msm is associated with changes in the cell envelope's structural integrity and increased sensitivity to toxic compounds, such as certain antibiotics and copper (Morita et al., 2006; Eagen et al., 2018). Rv0016c encodes PbpA, a transpeptidase involved in peptidoglycan crosslinking during cell division (Kieser and Rubin, 2014; Viswanathan et al., 2017), and PerM is a divisome component (Goodsmith et al., 2015; Wang et al., 2019). Rv2553 encodes a putative transglycosylase, and its Msm homolog localized to the septum and poles and interacted with FtsQ (Wu et al., 2018). We further analyzed underrepresented hits that encode integral membrane proteins (Figure 5 and Table 4). Six out of the 14 hits: $p b p A, \operatorname{per} M$, rodA, rv2553c, mmpS3/lamA, and $f h a B / f i p A$ encode cell division-related proteins (Sureka et al., 2010; Kieser and Rubin, 2014; Goodsmith et al., 2015; den Blaauwen et al., 2017; Rego et al., 2017; Viswanathan et al., 2017; Arora et al., 2018; Wang et al., 2019). The increased requirements of these genes when Rv0954 is missing supports our hypothesis that Rv0954 is involved in cell division or cell wall biosynthesis.

\section{Rv0954 Is Phosphorylated in Mtb}

The interaction between Rv0954 and PknH indicated that Rv0954 might undergo phosphorylation (Supplementary Figure 2). To test this hypothesis, we treated protein lysates with phosphatase and examined Rv0954's migration pattern by western blotting (Figure 6A). Rv0954 migrated as double bands without phosphatase treatment; incubation with alkaline phosphatase down-shifted both bands, indicating that Rv0954 may be subject to phosphorylation and additional modifications, such as acetylation, as previously reported (Xie et al., 2015). The control protein Rv0060 showed no alteration in migration patterns after the same treatment.

We found four phosphorylation sites in the C-terminus of Rv0954 by mass spectrometry: T257, S248, S256, and S264 (Figure 6B). We mutated all four sites to generate phosphoablative PA4 (Thr/Ser to Ala) or phospho-mimetic PD4 (Thr/Ser to Asp) mutants and evaluated the impact of phosphorylation on Rv0954. Both phospho mutants retained septal localization, either when expressed in addition to the WT MSMEG_5518 homologous allele (Figure 6C) or as the sole source of Rv0954 or MSMEG_5518 (Figure 6D). Our data indicate that Rv0954 
TABLE 2 | Drug susceptibility of H37Rv WT, Arv0954, and complemented strains.

\begin{tabular}{|c|c|c|c|c|c|}
\hline \multirow[t]{2}{*}{ Antibiotic } & \multirow{2}{*}{$\begin{array}{l}\text { Target biosynthetic } \\
\text { pathway }\end{array}$} & \multicolumn{3}{|c|}{$\mathrm{MIC}_{90}(\mu \mathrm{g} / \mathrm{ml})$} & \multirow[t]{2}{*}{ MICratio $^{a}$} \\
\hline & & WT & $\Delta r v 0954$ & comp & \\
\hline Ethambutol & $\begin{array}{l}\text { Arabinogalactan (Favrot } \\
\text { and Ronning, 2012) }\end{array}$ & 1.25 & 1.25 & 2.5 & 1 \\
\hline BTZ043 & $\begin{array}{l}\text { Arabinogalactan (Favrot } \\
\text { and Ronning, 2012) }\end{array}$ & 0.0025 & 0.0025 & 0.0025 & 1 \\
\hline Ethionamide & $\begin{array}{l}\text { Mycolic acid (Favrot and } \\
\text { Ronning, 2012) }\end{array}$ & 1 & 0.5 & 0.5 & 2 \\
\hline Delamanid & $\begin{array}{l}\text { Mycolic acid (Matsumoto } \\
\text { et al., 2006) }\end{array}$ & 0.5 & 0.25 & 0.5 & 2 \\
\hline Triclosan & $\begin{array}{l}\text { Mycolic acid (Favrot and } \\
\text { Ronning, 2012) }\end{array}$ & 37.5 & 75 & 37.5 & 0.5 \\
\hline Isoniazid & $\begin{array}{l}\text { Mycolic acid (Favrot and } \\
\text { Ronning, 2012) }\end{array}$ & 0.05 & 0.05 & 0.05 & 1 \\
\hline PA-824 & $\begin{array}{l}\text { Mycolic acid (Favrot and } \\
\text { Ronning, 2012) }\end{array}$ & 0.25 & 0.125 & 0.25 & 2 \\
\hline SQ109 & $\begin{array}{l}\text { Mycolic acid (Sacksteder } \\
\text { et al., 2012) }\end{array}$ & 0.625 & 0.625 & 0.625 & 1 \\
\hline Meropenem & $\begin{array}{l}\text { Peptidoglycan } \\
\text { (Goodsmith et al., 2015) }\end{array}$ & 10 & 5 & 10 & 2 \\
\hline D-cycloserine & $\begin{array}{l}\text { Peptidoglycan (Prosser } \\
\text { and de Carvalho, 2013) }\end{array}$ & 5 & 5 & 5 & 1 \\
\hline Vancomycin & $\begin{array}{l}\text { Peptidoglycan } \\
\text { (Goodsmith et al., 2015) }\end{array}$ & 10 & 5 & 10 & 2 \\
\hline
\end{tabular}

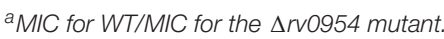

TABLE 3 | Underrepresented Tnseq hits in Arv0954 as compared with WT.

\begin{tabular}{lll}
\hline Rv\# & Gene & Annotation \\
\hline Rv0954 & & \\
Rv1252c & IprE & Probable lipoprotein \\
Rv2165c & & rRNA subunit methyltransferase H \\
Rv3215 & entC & Isochorismate synthase \\
Rv3593 & IpqF & Probable lipoprotein \\
Rv1159* & pimE & Mannosyltransferase \\
Rv0876c & & Transmembrane protein \\
Rv1080c & greA & Transcription elongation factor \\
Rv3267 & & Hypothetical protein \\
Rv0238 & & Transcription regulator \\
Rv3789 & & Integral membrane protein \\
Rv0949 & uvrD1 & ATP-dependent DNA helicase \\
Rv0016c* & pbpA & Penicillin-binding protein A \\
Rv0384c & clpB & Chaperone \\
Rv0955* & perM & Cell division protein (Goodsmith et al., 2015; Wang et al., 2019) \\
Rv2553c* & & Potential cell division protein (Wu et al., 2018) \\
Rv2507 & & Proline rich membrane protein \\
Rv1836c & & Hypothetical protein \\
Rv0211 & pckA & Phosphoenolpyruvate carboxykinase
\end{tabular}

Tnseq hits that meet the threshold of adjusted $p$-value $<0.01$ and $\log 2$ fold change $<-5$. The hits are ranked by log2 fold change from smallest to largest. The dataset is generated from two biological replicates. Annotations of each hit are adapted from PATRIC (https://www.patricbrc.org/) and FLUTE (http://orca2.tamu. edu/U19/) unless otherwise indicated. Genes involved in cell division or cell wall biosynthesis are marked with*

phosphorylation does not affect cell division in standard growth conditions yet may play a role in regulating cell division in specific, to be determined physiological conditions.

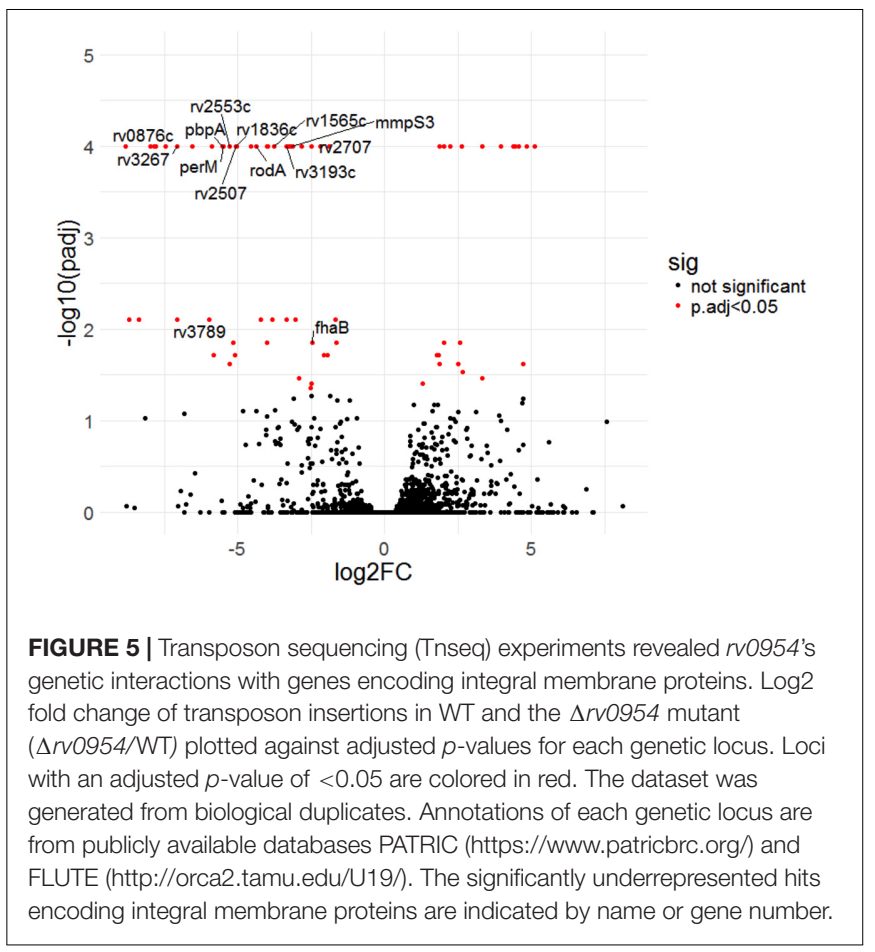

TABLE 4 | Underrepresented Tnseq hits in $\Delta$ rv0954 that encode integral membrane proteins.

\begin{tabular}{lccl}
\hline Rv\# & Gene & TMH & Annotation \\
\hline Rv0876c & & 10 & Hypothetical protein \\
Rv3267 & & 1 & Hypothetical protein \\
Rv3789 & & 4 & Integral membrane protein \\
Rv0016c & pbpA & 1 & Penicillin-binding protein A \\
Rv0955 & perM & 10 & Cell division protein (Goodsmith et al., 2015; \\
& & & Wang et al., 2019) \\
Rv2553c & & 1 & Potential cell division protein (Wu et al., 2018) \\
Rv2507 & & 1 & Proline rich membrane protein \\
Rv1836c & & 1 & Hypothetical protein \\
Rv0017c & rodA & 12 & Cell division protein RodA \\
Rv1565c & & 10 & Hypothetical protein \\
Rv3193c & & 7 & Hypothetical protein \\
Rv2198c & mmpS3/lamA & 1 & Cell division protein (Rego et al., 2017) \\
Rv2707 & & 6 & Hypothetical protein \\
Rv0019c & fhaB/fipA & 1 & Cell division protein (Sureka et al., 2010; Kieser \\
& & & and Rubin, 2014)
\end{tabular}

Tnseq hits that encode proteins with THMs and meet the threshold of adjusted $p$-value $<0.05$ and log2 fold change $<-2$. The hits are ranked by log 2 fold change from smallest to largest. The dataset is generated from two biological replicates. Annotations of each gene are adapted from PATRIC (https://www.patricbrc.org/) and FLUTE (http://orca2.tamu.edu/U19/).

\section{DISCUSSION}

There are several possible roles that Rv0954 could play during cell division. With four predicted TMHs and a stretched C-terminal domain, Rv0954 could serve as a structural scaffold to recruit additional division proteins. Protein co-localization experiments (Figures 1, 2) showed that Rv0954 lingers at mid-cell from before 
cytokinesis to cell constriction, possibly recruiting late division proteins such as LamA, which localizes to the mid-cell after FtsZ (Rego et al., 2017), and PbpA. Another possible role of Rv0954 is to anchor intracellular divisome components to the inner cell membrane, similar to the role of ZipA in E. coli. In fact, like the proline/glutamine-rich intracellular tail of Rv0954, the cytoplasmic domain of ZipA contains a region that is made of $31 \%$ proline and 23\% glutamine (Hale and de Boer, 1997). This region may form a long rigid linker to protrude into the cytoplasm for some distance and is proposed to facilitate interaction with FtsZ (Hale and de Boer, 1997). Mycobacteria lack homologs of ZipA, and Rv0954 is unlikely to fill the same role given that FtsZ localizes to the mid cell prior to Rv0954. However, it is plausible that a cytoplasmic division protein other than FtsZ needs membrane attachment via Rv0954.

Rv0954 is phosphorylated in Mtb, possibly by its interaction partner PknH (Figure 3, Table 1, and Supplementary Figure 2). Phosphorylation of division proteins is a major mechanism for mycobacteria to regulate cell division (Kieser and Rubin, 2014; Baranowski et al., 2019). Protein phosphorylation may alter its activity, localization, or interaction with other proteins. The Mtb phospho mutants did not display morphological abnormalities and Rv0954 accumulated at the mid-cell with and without phosphorylation (Figure 6), suggesting that phosphorylation may play an accessory role during normal growth. Alternative explanations include additional phosphorylation sites that exist within Rv0954 or phenotypes masked by proteins with overlapping functions.

It is common to find redundancies within the bacterial divisome (de Boer, 2010; Kieser and Rubin, 2014). E. coli, for instance, expresses ZipA and FtsA that have overlapping roles in membrane attachment of FtsZ; the Z-ring could form when either ZipA or FtsA was present, but not when both were inactivated (Pichoff and Lutkenhaus, 2002). Functional redundancy may explain $\Delta r v 0954$ 's lack of phenotypes in cell morphology, drug susceptibility, and growth in acidic $\mathrm{pH}$ or low magnesium. These results are in contrast to the phenotypes observed when perM, which is part of the same operon, was deleted (Goodsmith et al., 2015; Wang et al., 2019). Although we cannot exclude the possibility that rv0954 may have an essential role in vivo, our current data did not motivate infection experiments. Comparing transposon insertion profiles of the $\Delta r v 0954$ mutant to WT Mtb could help reveal overlapping pathways. We detected a reduced number of insertions in several genes that encode integral membrane proteins (Table 4). These include six related to cell division and eight of unknown functions. Future studies of these hits might reveal additional mycobacterial division factors and unmask Rv0954's functions.

Two cell division genes, $p b p A$ and $\operatorname{lam} A$, contained fewer transposon insertions in bacteria lacking Rv0954 than WT. PbpA and LamA also physically interacted with Rv0954 in the Co-IP experiment (Figure 3 and Table 1). PbpA is a transpeptidase
A

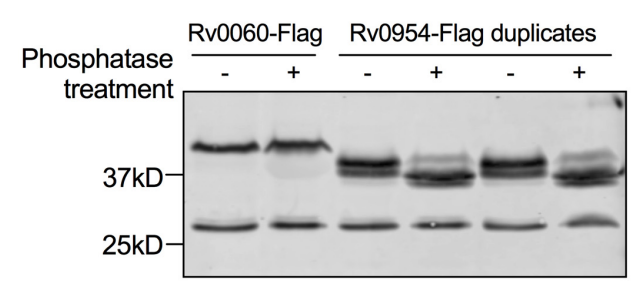

C

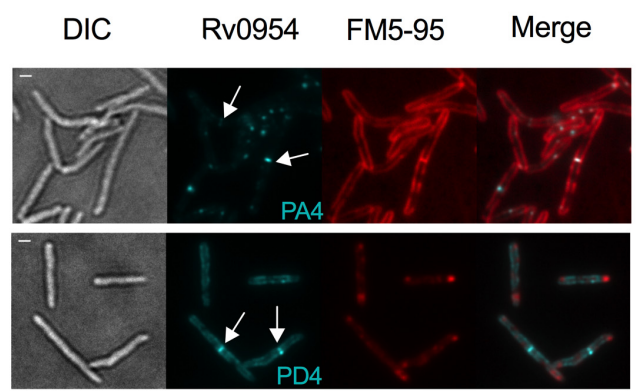

B

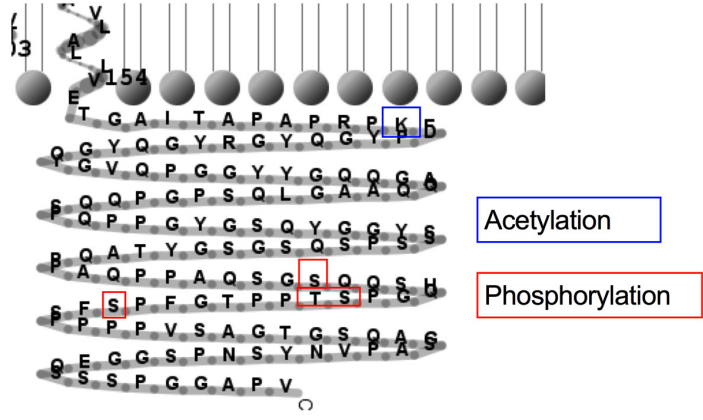

D

DIC Rv0954 FM5-95 Merge

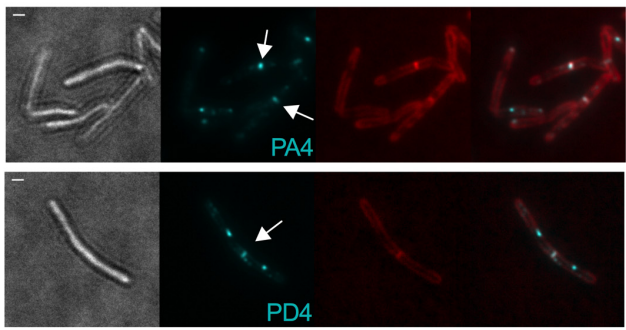

FIGURE 6 | Rv0954 is phosphorylated. (A) Detection of Rv0060-Flag (42 kD) and Rv0954-Flag (34 kD) in Mtb whole-cell protein lysates after treatment or not with alkaline phosphatase. The western blot was probed with an anti-Flag antibody. Data are representative of two independent experiments. (B) Schematic of the previously reported acetylation (Xie et al., 2015) and newly discovered phosphorylation sites in the C-terminus of Rv0954. (C,D) Representative microscopy images

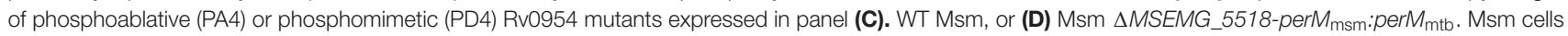
were collected and stained with membrane dye FM5-95 before imaging. Arrows point to the mid-cell localization of Rv0954. Scale bar, $1 \mu \mathrm{m}$. 
that synthesizes the septal peptidoglycan; cells lacking $\mathrm{PbpA}$ were hypersusceptible to $\beta$-lactams (Arora et al., 2018). PbpA interacted with FhaA and $\operatorname{CrgA}$, both are related to peptidoglycan biosynthesis and cell shape maintenance (Plocinski et al., 2011; Viswanathan et al., 2017). LamA is a highly conserved divisome component across mycobacterial species. It inhibits growth at the new cell poles and creates asymmetry. When treated with cell wall-targeting antibiotics such as rifampicin, cells lacking LamA were killed more uniformly and faster (Rego et al., 2017; Logsdon and Aldridge, 2018). Rv0954 mutant did not show increased sensitivity to cell wall-targeting compounds (Table 2), likely because of the functional overlap among divisome components and the complexity in cell division regulation. Future work on the mechanisms by which Rv0954 interacts with PbpA and LamA could help us understand how mycobacteria integrate growth and cell cycle and prioritize antitubercular targets.

The mycobacterial cell wall is the first barrier that protects bacteria from host stresses and antibiotics. Maintenance of cell wall integrity requires properly regulated cell division. Our genetic and biochemical study of a previously uncharacterized protein Rv0954, including its mid-cell localization, physical interactors, mutant phenotypes, and protein modifications, supported Rv0954 as a new component of the mycobacterial divisome.

\section{DATA AVAILABILITY STATEMENT}

The data presented in the study are deposited in the NCBI repository, accession number PRJNA768285; https://www.ncbi. nlm.nih.gov/bioproject/PRJNA678285.

\section{REFERENCES}

Abramoff, M., Magalhães, P., and Ram, S. J. (2003). Image Processing with ImageJ. Biophotonics Int. 11, 36-42.

Aldridge, B. B., Fernandez-Suarez, M., Heller, D., Ambravaneswaran, V., Irimia, D., Toner, M., et al. (2012). Asymmetry and aging of mycobacterial cells lead to variable growth and antibiotic susceptibility. Science 335, 100-104. doi: 10. $1126 /$ science. 1216166

Arora, D., Chawla, Y., Malakar, B., Singh, A., and Nandicoori, V. K. (2018). The transpeptidase PbpA and noncanonical transglycosylase RodA of Mycobacterium tuberculosis play important roles in regulating bacterial cell lengths. J. Biol. Chem. 293, 6497-6516. doi: 10.1074/jbc.M117.81 1190

Baranowski, C., Rego, E. H., and Rubin, E. J. (2019). The Dream of a Mycobacterium. Microbiol. Spectr. 7, G3-G0008. doi: 10.1128/microbiolspec. GPP3-0008-2018

Botella, H., Yang, G., Ouerfelli, O., Ehrt, S., Nathan, C. F., and Vaubourgeix, J. (2017). Distinct Spatiotemporal Dynamics of Peptidoglycan Synthesis between Mycobacterium smegmatis and Mycobacterium tuberculosis. MBio 8, e0118317. doi: $10.1128 / \mathrm{mBio} .01183-17$

Cameron, T., Zupan, J., and Zambryski, P. (2015). The essential features and modes of bacterial polar growth. Trends Microbiol. 23, 347-353. doi: 10.1016/j.tim. 2015.01.003

Crellin, P. K., Kovacevic, S., Martin, K. L., Brammananth, R., Morita, Y. S., BillmanJacobe, H., et al. (2008). Mutations in pimE Restore Lipoarabinomannan Synthesis and Growth in a Mycobacterium smegmatis lpqW Mutant. J. Bacteriol. 190, 3690-3699. doi: 10.1128/JB.00200-08

de Boer, P. A. J. (2010). Advances in understanding E. coli cell fission. Curr. Opin. Microbiol. 13, 730-737. doi: 10.1016/j.mib.2010.09.015

\section{AUTHOR CONTRIBUTIONS}

RW designed and performed experiments, analyzed data, and drafted the manuscript. SE supervised the project, acquired funding, and reviewed and edited the manuscript. Both authors contributed to the article and approved the submitted version.

\section{FUNDING}

This work was supported by NIH grants U19 AI111143 and P01 AI143575.

\section{ACKNOWLEDGMENTS}

We thank K. G. Papavinasasundaram (University of Massachusetts) and J. Fernandez (Rockefeller University) for LCMS/MS analyses. We thank A. North (Rockefeller University) for microscopy training and advice. We also thank M. Whitaker and S. Schrader for carefully editing the first draft. L. Chan and C. Harris helped with molecular cloning. Figure 3 was created with https://flourish.studio/.

\section{SUPPLEMENTARY MATERIAL}

The Supplementary Material for this article can be found online at: https://www.frontiersin.org/articles/10.3389/fmicb. 2021.626461/full\#supplementary-material

DeJesus, M. A., Gerrick, E. R., Xu, W., Park, S. W., Long, J. E., Boutte, C. C., et al. (2017). Comprehensive Essentiality Analysis of the Mycobacterium tuberculosis Genome via Saturating Transposon Mutagenesis. MBio 8, e0213316. doi: $10.1128 / \mathrm{mBio} .02133-16$

den Blaauwen, T., Hamoen, L. W., and Levin, P. A. (2017). The divisome at 25: the road ahead. Curr. Opin. Microbiol. 36, 85-94. doi: 10.1016/j.mib.2017. 01.007

Eagen, W. J., Baumoel, L. R., Osman, S. H., Rahlwes, K. C., and Morita, Y. S. (2018), Deletion of PimE mannosyltransferase results in increased copper sensitivity in Mycobacterium smegmatis. FEMS Microbiol. Lett. 365:29390083. doi: 10.1093/ femsle/fny025

Favrot, L., and Ronning, D. R. (2012). Targeting the mycobacterial envelope for tuberculosis drug development. Expert Rev. Anti Infec. Ther. 10, 1023-1036. doi: $10.1586 /$ eri.12.91

Gee, C. L., Papavinasasundaram, K. G., Blair, S. R., Baer, C. E., Falick, A. M., King, D. S., et al. (2012). A phosphorylated pseudokinase complex controls cell wall synthesis in mycobacteria. Sci. Signal. 5:ra7. doi: 10.1126/scisignal.2002525

Goodsmith, N., Guo, X. V., Vandal, O. H., Vaubourgeix, J., Wang, R., Botella, H., et al. (2015). Disruption of an M. tuberculosis Membrane Protein Causes a Magnesium-dependent Cell Division Defect and Failure to Persist in Mice. PLoS Pathog. 11:e1004645. doi: 10.1371/journal.ppat.1004645

Hale, C. A., and de Boer, P. A. (1997). Direct binding of FtsZ to ZipA, an essential component of the septal ring structure that mediates cell division in E. coli. Cell 88, 175-185. doi: 10.1016/s0092-8674(00)81838-3

Hirota, Y., Ryter, A., and Jacob, F. (1968). Thermosensitive mutants of E. coli affected in the processes of DNA synthesis and cellular division. Cold Spring Harb. Symp. Quant. Biol. 33, 677-693. doi: 10.1101/sqb.1968.033.01.077

Kieser, K. J., and Rubin, E. J. (2014). How sisters grow apart: mycobacterial growth and division. Nat. Rev. Microbiol. 12, 550-562. doi: 10.1038/nrmicro3299 
Logsdon, M. M., and Aldridge, B. B. (2018). Stable Regulation of Cell Cycle Events in Mycobacteria: insights From Inherently Heterogeneous Bacterial Populations. Front. Microbiol. 9:514. doi: 10.3389/fmicb.2018. 00514

Marmiesse, M., Brodin, P., Buchrieser, C., Gutierrez, C., Simoes, N., Vincent, V., et al. (2004). Macro-array and bioinformatic analyses reveal mycobacterial "core" genes, variation in the ESAT-6 gene family and new phylogenetic markers for the Mycobacterium tuberculosis complex. Microbiology 150, 483-496. doi: $10.1099 /$ mic. $0.26662-0$

Matsumoto, M., Hashizume, H., Tomishige, T., Kawasaki, M., Tsubouchi, H., Sasaki, H., et al. (2006). OPC-67683, a Nitro-Dihydro-Imidazooxazole Derivative with Promising Action against Tuberculosis In Vitro and In Mice. PLoS Med. 3:e466. doi: 10.1371/journal.pmed.0030466

Meniche, X., Otten, R., Siegrist, M. S., Baer, C. E., Murphy, K. C., Bertozzi, C. R., et al. (2014). Subpolar addition of new cell wall is directed by DivIVA in mycobacteria. Pro. Natl. Acad. Sci. U. S. A. 111, E3243-E3251. doi: 10.1073/ pnas. 1402158111

Morita, Y. S., Sena, C. B. C., Waller, R. F., Kurokawa, K., Sernee, M. F., Nakatani, F., et al. (2006). PimE is a polyprenol-phosphate-mannose-dependent mannosyltransferase that transfers the fifth mannose of phosphatidylinositol mannoside in mycobacteria. J. Biol. Chem. 281, 25143-25155. doi: 10.1074/jbc. M604214200

Pichoff, S., and Lutkenhaus, J. (2002). Unique and overlapping roles for ZipA and FtsA in septal ring assembly in Escherichia coli. EMBO J. 21, 685-693. doi: 10.1093/emboj/21.4.685

Plocinski, P., Ziolkiewicz, M., Kiran, M., Vadrevu, S. I., Nguyen, H. B., Hugonnet, J., et al. (2011). Characterization of CrgA, a new partner of the Mycobacterium tuberculosis peptidoglycan polymerization complexes. J. Bacteriol. 193, 32463256. doi: 10.1128/JB.00188-11

Prosser, G. A., and de Carvalho, L. P. S. (2013). Metabolomics Reveal d-Alanine:dAlanine Ligase As the Target of d-Cycloserine in Mycobacterium tuberculosis. ACS Med. Chem. Lett. 4, 1233-1237. doi: 10.1021/ml400349n

Rego, E. H., Audette, R. E., and Rubin, E. J. (2017). Deletion of a mycobacterial divisome factor collapses single-cell phenotypic heterogeneity. Nature 546, 153-157. doi: 10.1186/s40945-017-0033-9.Using

Sacksteder, K. A., Protopopova, M., Barry, C. E., Andries, K., and Nacy, C. A. (2012). Discovery and development of SQ109: a new antitubercular drug with a novel mechanism of action. Future Microbiol. 7, 823-837. doi: 10.2217/fmb. 12.56

Santi, I., Dhar, N., Bousbaine, D., Wakamoto, Y., and McKinney, J. D. (2013). Single-cell dynamics of the chromosome replication and cell division cycles in mycobacteria. Nat. Commun. 4:2470. doi: 10.1038/ncomms3470
Sharma, K., Gupta, M., Pathak, M., Gupta, N., Koul, A., Sarangi, S., et al. (2006). Transcriptional control of the mycobacterial embCAB operon by $\mathrm{PknH}$ through a regulatory protein, EmbR, in vivo. J. Bacteriol. 188, 2936-2944. doi: 10.1128/JB.188.8.2936-2944.2006

Sureka, K., Hossain, T., Mukherjee, P., Chatterjee, P., Datta, P., Kundu, M., et al. (2010). Novel Role of Phosphorylation-Dependent Interaction between FtsZ and FipA in Mycobacterial Cell Division. PLoS One 5:e8590. doi: 10.1371/ journal.pone. 0008590

Viswanathan, G., Yadav, S., Joshi, S. V., and Raghunand, T. R. (2017). Insights into the function of FhaA, a cell division-associated protein in mycobacteria. FEMS Microbiol. Lett.364:28013239. doi: 10.1093/femsle/fnw294

Wang, R., Kreutzfeldt, K., Botella, H., Vaubourgeix, J., Schnappinger, D., and Ehrt, S. (2019). Persistent Mycobacterium tuberculosis infection in mice requires PerM for successful cell division. ELife 8:e49570. doi: 10.7554/eLife.49570

Williamson, M. P. (1994). The structure and function of proline-rich regions in proteins. Biochem. J. 297, 249-260. doi: 10.1042/bj2970249

Wu, K. J., Zhang, J., Baranowski, C., Leung, V., Rego, E. H., Morita, Y. S., et al. (2018). Characterization of Conserved and Novel Septal Factors in Mycobacterium smegmatis. J. Bacteriol. 200:29311277. doi: 10.1128/JB. 00649-17

Xie, L., Wang, X., Zeng, J., Zhou, M., Duan, X., Li, Q., et al. (2015). Proteomewide lysine acetylation profiling of the human pathogen Mycobacterium tuberculosis. Int. J. Biochem. Cell Biol. 59, 193-202. doi: 10.1016/j.biocel.2014. 11.010

Xu, W., DeJesus, M. A., Rücker, N., Engelhart, C. A., Wright, M. G., Healy, C., et al. (2017). Chemical Genetic Interaction Profiling Reveals Determinants of Intrinsic Antibiotic Resistance in Mycobacterium tuberculosis. Antimicrob. Agents Chemother. 61, e01334-17. doi: 10.1128/AAC.01334-17

Zheng, X., Papavinasasundaram, K. G., and Av-Gay, Y. (2007). Novel substrates of Mycobacterium tuberculosis PknH Ser/Thr kinase. Biochem. Biophys. Res. Commun. 355, 162-168. doi: 10.1016/j.bbrc.2007.01.122

Conflict of Interest: The authors declare that the research was conducted in the absence of any commercial or financial relationships that could be construed as a potential conflict of interest.

Copyright $(2021$ Wang and Ehrt. This is an open-access article distributed under the terms of the Creative Commons Attribution License (CC BY). The use, distribution or reproduction in other forums is permitted, provided the original author(s) and the copyright owner(s) are credited and that the original publication in this journal is cited, in accordance with accepted academic practice. No use, distribution or reproduction is permitted which does not comply with these terms. 